\title{
MR imaging in discriminating between benign and malignant paediatric ovarian masses: a systematic review
}

\author{
Lotte W. E. van Nimwegen ${ }^{1}$ - Annelies M. C. Mavinkurve-Groothuis ${ }^{1}$ (D) - Ronald R. de Krijger ${ }^{1,2} \cdot$ Caroline C. C. Hulsker $^{1}$ • \\ Angelique J. Goverde ${ }^{3}$ • József Zsiros ${ }^{1}$ • Annemieke S. Littooij ${ }^{1,4}$
}

Received: 6 June 2019 / Revised: 3 August 2019 / Accepted: 8 August 2019 / Published online: 16 September 2019

(C) The Author(s) 2019

\begin{abstract}
Objectives The use of magnetic resonance (MR) imaging in differentiation between benign and malignant adnexal masses in children and adolescents might be of great value in the diagnostic workup of sonographically indeterminate masses, since preserving fertility is of particular importance in this population. This systematic review evaluates the diagnostic value of MR imaging in children with an ovarian mass.

Methods The review was made according to the PRISMA Statement. PubMed and EMBASE were systematically searched for studies on the use of MR imaging in differential diagnosis of ovarian masses in both adult women and children from 2008 to 2018.

Results Sixteen paediatric and 18 adult studies were included. In the included studies, MR imaging has shown good diagnostic performance in differentiating between benign and malignant ovarian masses. MR imaging techniques including diffusionweighted imaging (DWI) and dynamic contrast-enhanced (DCE) imaging seem to further improve the diagnostic performance. Conclusion The addition of DWI with apparent diffusion coefficient (ADC) values measured in enhancing components of solid lesions and DCE imaging may further increase the good diagnostic performance of MR imaging in the pre-operative differentiation between benign and malignant ovarian masses by increasing specificity. Prospective age-specific studies are needed to confirm the high diagnostic performance of MR imaging in children and adolescents with a sonographically indeterminate ovarian mass.

Key Points

- MR imaging, based on several morphological features, is of good diagnostic performance in differentiating between benign and malignant ovarian masses. Sensitivity and specificity varied between 84.8 to $100 \%$ and 20.0 to $98.4 \%$, respectively.

- MR imaging techniques like diffusion-weighted imaging (DWI) and dynamic contrast-enhanced (DCE) imaging seem to improve the diagnostic performance.

- Specific studies in children and adolescents with ovarian masses are required to confirm the suggested increased diagnostic performance of DWI and DCE in this population.
\end{abstract}

Keywords Ovarian neoplasms $\cdot$ Magnetic resonance imaging $\cdot$ Systematic review

Electronic supplementary material The online version of this article (https://doi.org/10.1007/s00330-019-06420-4) contains supplementary material, which is available to authorized users.

Annelies M. C. Mavinkurve-Groothuis

a.m.c.mavinkurve-groothuis@ @ prinsesmaximacentrum.nl

1 Princess Máxima Center for Pediatric Oncology, Heidelberglaan 25, 3584, CS Utrecht, The Netherlands
2 Department of Pathology, University Medical Center Utrecht, Utrecht, The Netherlands

3 Department of Reproductive Medicine and Gynaecology, University Medical Center of Utrecht, Utrecht, The Netherlands

4 Department of Radiology and Nuclear Medicine, University Medical Center Utrecht, Utrecht, The Netherlands 


\section{List of abbreviations}

ADC Apparent diffusion coefficient

AUC Area under the curve

DCE Dynamic contrast-enhanced imaging

DWI Diffusion-weighted imaging

ESUR European Society of Urogenital Radiology

IOTA International Ovarian Tumor Analysis

MR Magnetic resonance

MRE\% Maximum relative enhancement percentage

NPV Negative predictive value

PPV Positive predictive value

ROI Regions of interest

$\mathrm{SI}_{60} \quad$ Signal intensity at $60 \mathrm{~s}$ after enhancement

SImax Maximum absolute enhancement

SIrel Maximum relative enhancement

THR Time of half rising

TICs Time-intensity curves

$\mathrm{TTP}_{200}$ Time to peak within $200 \mathrm{~s}$ after enhancement

\section{Introduction}

Ovarian malignancies in children and adolescents are relatively rare, with an incidence of 3 per 100,000 compared with 56 cases per 100,000 at the age of 65 to 69 years [1-3]. Despite this low incidence, ovarian tumours constitute the most common gynaecological malignancy in children and adolescents. Paediatric ovarian masses encompass a variety of benign and malignant tumours, including rare types such as sex cordstromal tumours [4-6]. Both this heterogeneity and the importance of fertility preservation in this age group make the diagnostic assessment of these masses challenging.

While malignant ovarian neoplasms may need a more aggressive surgical approach, benign masses can either be safely monitored or undergo simple resection allowing for a fertilityand ovary-sparing approach [7]. Being able to discriminate between benign and malignant masses of the ovary is therefore of considerable clinical importance in the initial surgical management $[4,8]$. Ultrasound is the first imaging modality in the diagnostic assessment of ovarian masses at any age. Clinically useful rules have been established by the International Ovarian Tumor Analysis (IOTA) group to differentiate between benign and malignant masses. Nevertheless, in about one-fifth of the cases, the nature of the ovarian mass remains undefined [9].

In case of sonographically indeterminate ovarian masses, magnetic resonance (MR) imaging can provide additional information, e.g. on the different components of the mass, tumour rupture and peritoneal depositions. Figures 1 and 2 show examples of an immature teratoma grade I (treated as a benign tumour with local resection and follow-up) and a malignant yolk sac tumour. Functional imaging techniques like diffusion-weighted imaging (DWI) and dynamic contrast- enhanced (DCE) imaging could be of additional value [10]. DCE enables qualitative, quantitative or semi-quantitative evaluation of tumour vascularity, thereby providing information about the nature of the mass. This investigation is based on enhancement patterns, expressed as time-intensity curves (TICs), of which three different types are acknowledged. Type I displays a gradual, continuous rise in signal intensity; type II shows a moderate rise in signal intensity followed by a plateau; and type III is characterised as early washout $[11,12]$. In adults, several studies have evaluated the diagnostic value of MR imaging in differentiating between malignant and benign neoplasms and characterising the specific nature of ovarian masses. Based on these studies, the European Society of Urogenital Radiology (ESUR) has developed an algorithmic approach for the imaging of the sonographically indeterminate adnexal mass [7, 13-16]. However, data on the role of MR imaging in discriminating between benign and malignant ovarian masses in children is scarce. In this systematic review, we evaluate the diagnostic value of MR imaging in children and adolescents with an ovarian mass, including the value of additional MR techniques.

\section{Methods}

\section{Search strategy and eligibility criteria}

This review is written according to the PRISMA Statement [17]. A thorough search of PubMed and EMBASE for all available literature published from 2008 to 2018 was performed. These libraries were systematically searched for original studies on the use of MR imaging in differential diagnosis of ovarian masses in both adult women and children. We classified studies into two groups. Studies were classified as 'paediatric', when the age of all included patients was 18 years or less. Studies performed on adult women, on the other hand, were classified as 'adult'. The full search strategy is provided in Supplementary Table 1. Articles were included if suspected ovarian masses were evaluated with MR imaging (either $1.5 \mathrm{~T}$ or $3.0 \mathrm{~T}$ ), including the evaluation of contrast enhancement, and were compared with a histopathology reference standard. Studies providing no description of MR imaging findings and studies on adult women that analysed selectively benign, borderline or malignant masses were excluded. However, similar studies as well as case reports performed on paediatric patients were included, in order to minimise the risk of missing relevant studies. Since ovarian carcinomas are very rare in children, only studies performed on adult patients that included more than $20 \%$ of malignant tumours other than carcinoma were considered relevant for this review. This particular cutoff was chosen pragmatically, since it was expected most MR studies in adult ovarian tumours focus on epithelial neoplasms, due to its prevalence of $80-90 \%$. 
Fig. 1 An example of immature teratoma grade 1 of the right ovary in a 15 -year-old girl, treated as a benign tumour with local resection and follow-up. Axial T1-weighted before and after administration of gadolinium contrast (a, c), axial T1-weighted with fat-suppression (b) and sagittal T2-weighted turbo spin echo (d) show a cystic-solid mass with fatty components (arrows). Intralesional fat is diagnostic for a teratoma. The relative large amount of enhancing parts increases the risk of immature components
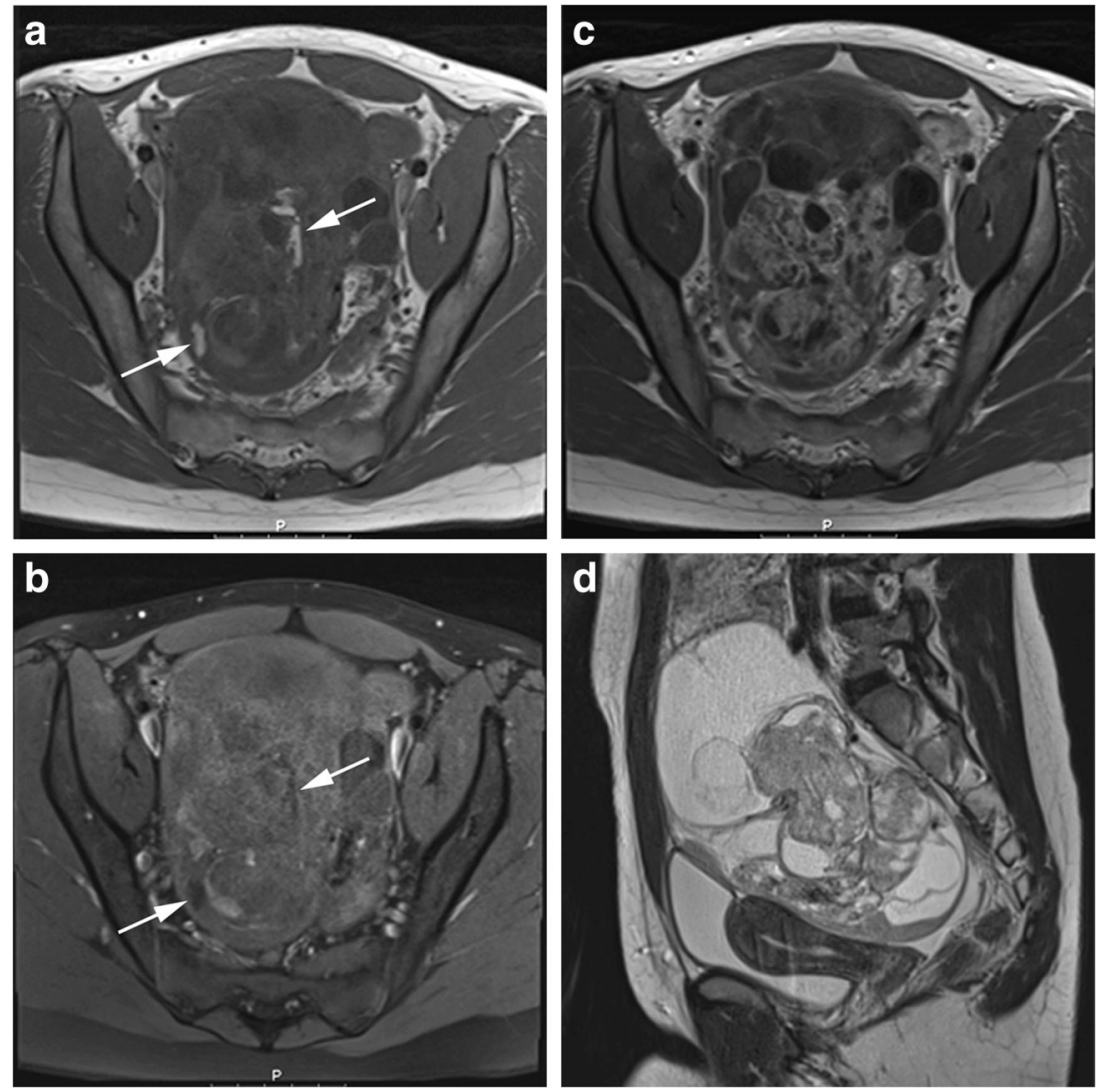

All studies resulting from the literature search were assessed independently by two researchers (A.M., L.N.). Disagreements about study inclusion or exclusion were settled by consensus.

\section{Quality assessment}

The quality of the individual studies was judged using the "Standards for Reporting Diagnostic Accuracy 2015" (STARD 2015) checklist [18]. Included studies were further assessed for methodologic quality independently by two researchers (A.M., L.N.), using the Oxford Centre for EvidenceBased Medicine Levels of Evidence Classification rubric [19].

\section{Data extraction}

From the included studies, population size expressed as the number of ovarian masses analysed, mean age of the participating patients, histopathological classification of the ovarian masses and MR imaging protocol and analysis, as well as MR imaging features of the concerning ovarian masses, were scored. As for MR imaging features, information about the following parameters were extracted: size, shape, boundary, wall and septum thickness, vegetation, mass configuration, bilaterality, signal intensity of T1-weighted imaging, ascites/pelvic fluid, peritoneal implants/nodules and contrast enhancement. If available, information on $b$-values used in DWI and apparent diffusion coefficient (ADC) values were collected. Concerning semi-quantitative DCE, data on TICs, enhancement amplitude and time to peak were included. Lastly, data on diagnostic performance expressed as sensitivity, specificity, accuracy, positive predictive value (PPV), negative predictive value (NPV) or area under the curve (AUC) for these individual parameters were extracted when provided.

\section{Results}

\section{Search strategy and eligibility criteria}

The study selection process is shown in Fig. 3. The search in PubMed and EMBASE resulted in 3015 studies, of which 536 studies turned out to be duplicates. The remaining 2479 studies were screened by title and abstract, based on which 2341 studies were excluded. Consequently, 138 articles were of potential relevance to this systematic review and their full texts were analysed. This led to the exclusion of another 104 studies. The remaining 34 studies were analysed in this review. 
Fig. 2 An example of yolk sac tumour of the right ovary in a 16year-old girl. Sagittal T2-

weighted turbo spin echo TSE (a) and T1-weighted gradient echo with fat suppression before and after administration of gadolinium contrast $(\mathbf{b}, \mathbf{c})$ show a large cystic solid mass in the lower abdomen. The enhancing parts of the lesion show relative impeded diffusion (arrow) at axial DWI (b1000 and ADC map; d,e)


\section{Quality assessment}

The studies in adult women were predominantly scored as Oxford Evidence level 2 (cross-sectional studies with consistently applied reference standard and blinding). Levels of evidence of the individual studies can be found in Table 1 . Quality assessment of the included studies in adult women, using the STARD 2015, is provided in Supplementary Table 2.

Since most studies in children and adolescents concerned either case reports or case series, the majority of these were scored as Oxford Evidence level 4, with the exception of two studies (one cross-sectional study, one non-consecutive study) (Table 1).

\section{Characteristics of included studies}

The characteristics of the included studies (18 'adult' $[11,12,20-35]$ and 16 'paediatric' studies [36-51]) are provided in Table 1. The mean age of patients included was 10.8 years in the paediatric and 46.9 years in the adult studies. The number of ovarian lesions analysed ranged between 1 and 74 in the paediatric studies and between 23 and 235 in the adult studies. All studies analysed the use of MR imaging in differentiating between benign and malignant tumours of the ovary, with several studies incorporating the differentiation of epithelial borderline tumours as well.

\section{Paediatric studies}

Table 2 shows MR imaging findings of the sixteen studies that were included: three cohort studies and 13 case reports. All three cohort studies analysed the diagnostic performance of MR imaging in children and adolescents with ovarian masses (or ovarian germ cell tumours specifically). The thirteen case reports describe limited data on MR characteristics. 
Fig. 3 The flowchart summarises the search process with the number of studies included and excluded

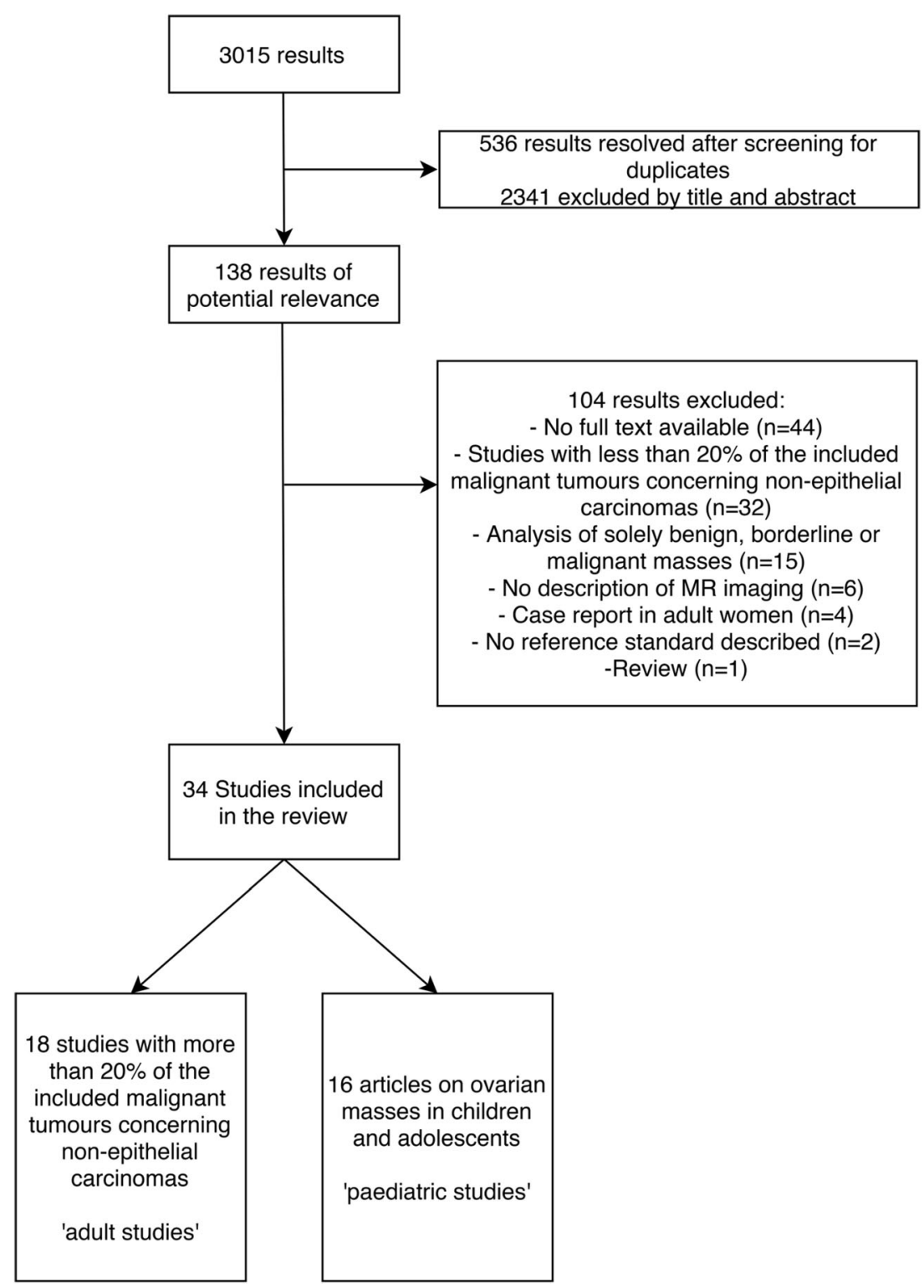

\section{Adult studies}

\section{MR imaging}

Ten studies provided a description of MR imaging features. The most often-described features ( $>4$ out of 10 studies) concerned size, thickness of walls and septa (when present), presence of vegetation, mass configuration, bilaterality, signal intensity on T2-weighted imaging, presence of ascites or peritoneal implants and contrast enhancement. An increased risk of malignancy was related to increased size of the lesion, increased wall thickness, presence and increased size of vegetation, mixed cystic and solid configuration, intermediate to high intensity on $\mathrm{T} 2$-weighted imaging, presence of contrast enhancement and of ascites or peritoneal implants.
Six of the studies performed an analysis of the diagnostic performance of MR imaging [23, 27, 29-31, 34]. Criteria predictive of malignancy, sensitivity, specificity, PPV, NPV and accuracy, if provided, are depicted in Table 3. Sensitivity and specificity, depending on the criteria used, varied between 84.8 to $100 \%$ and 20.0 to $98.4 \%$, respectively.

\section{DWI-MR imaging}

Eight studies investigated the value of DWI-MRI in the differential diagnosis of ovarian masses [20, 21, 23-26, $31,34]$. $b$-values $\left(\mathrm{s} / \mathrm{mm}^{2}\right)$, regions of interest (ROI) used to calculate ADC values $\left(\times 10^{-3} \mathrm{~mm}^{2} / \mathrm{s}\right)$ and diagnostic performance are shown in Table 4. Mean ADC values for benign and malignant lesions exhibited a 
Table 1 Characteristics of the studies included in this systematic review regarding the use of MR imaging in differential diagnosis of ovarian masses

\begin{tabular}{|c|c|c|c|c|}
\hline Study (reference) & $\begin{array}{l}\text { Oxford } \\
\text { level }\end{array}$ & $n$ & Mean age in years & $\begin{array}{l}\text { Histopathological classification of included ovarian } \\
\text { masses }\end{array}$ \\
\hline \multicolumn{5}{|l|}{ Adult studies } \\
\hline Li et al 2017 [11] & 2 & 102 & 57 (benign), 37 (borderline), 54 (malignant) & Benign $(n=15)$, borderline $(n=16)$, malignant $(n=71)$ \\
\hline Li et al 2015 [12] & 3 & 48 & NA (range 11-79) & Benign $(n=13)$, malignant $(n=35)$ \\
\hline Zhao et al 2018 [20] & 3 & 42 & 52 (benign), 41 (malignant) & Benign $(n=29)$, malignant $(n=13)$ \\
\hline Zhang et al 2012 [21] & 2 & 139 & 52 & $\begin{array}{l}\text { Cysts }(n=21), \text { endometriomas }(n=33), \\
\text { benign }(n=43), \text { malignant }(n=42)\end{array}$ \\
\hline Bernardin et al 2012 [22] & 2 & 67 & 48 & Benign $(n=31)$, malignant $(n=36)$ \\
\hline Nasr et al 2014 [23] 3 & & 23 & 36 (benign), 45 (malignant) & Benign $(n=12)$, malignant $(n=11)$ \\
\hline Takeuchi et al 2009 [24] & 2 & 49 & 59 & Benign $(n=10)$, borderline $(n=6)$, malignant $(n=33)$ \\
\hline Mansour et al 2015 [25] & 2 & 235 & 39 & Benign $(n=75)$, malignant $(n=160)$ \\
\hline Zhang et al 2012 [26] & 3 & 202 & 57 & Benign $(n=74)$, malignant $(n=128)$ \\
\hline Tsili et al 2008 [27] & 2 & 89 & 67 & Benign $(n=66)$, malignant $(n=23)$ \\
\hline Dilks et al 2010 [28] & 2 & 26 & 43 & Benign $(n=14)$, malignant $(n=12)$ \\
\hline Tsuboyama et al 2014 [29] & 2 & 127 & 53 & $\begin{array}{l}\text { Benign }(n=30) \text {, borderline }(n=31), \\
\quad \text { malignant }(n=66)\end{array}$ \\
\hline Elzayat et al 2017 [30] & 3 & 32 & 39 (benign), 34 (borderline), 43 (malignant) & Benign $(n=7)$, borderline $(n=4)$, malignant $(n=21)$ \\
\hline Emad-Eldin et al 2018 [31] & 2 & 65 & 44 & Benign $(n=30)$, borderline $(n=7)$, malignant $(n=28)$ \\
\hline Mansour et al 2015 [32] & 2 & 150 & 29 (benign), 39 (borderline), 46 (malignant) & Benign $(n=42)$, borderline $(n=26)$, malignant $(n=82)$ \\
\hline Li et al 2018 [33] & 2 & 109 & 57 (benign), 34 (borderline), 51 (malignant) & Benign $(n=15)$, borderline $(n=28)$, malignant $(n=66)$ \\
\hline Zhang et al 2014 [34] & 2 & 144 & $\begin{array}{l}37 \text { years (endometric cysts), } 40 \text { years } \\
\text { (teratomas) }\end{array}$ & Endometric cysts $(n=35)$, teratomas $(n=28)$ \\
\hline Zhao et al 2014 [35] & 2 & 50 & 51 (benign), 41 (borderline) & Benign $(n=26)$, borderline $(n=24)$ \\
\hline \multicolumn{5}{|l|}{ Paediatric studies } \\
\hline Emil et al 2017 [36] & 3 & 18 & 15 & Benign \\
\hline Marro et al 2016 [37] & 2 & 32 & 13 & Benign, borderline and malignant \\
\hline Thomas et al 2012 [38] & 4 & 1 & 14 & Bilateral mucinous cystadenomas \\
\hline Willems et al 2012 [39] & 4 & 1 & 15 & Benign mucinous cystadenoma \\
\hline Park et al $2010[40]$ & 4 & 1 & 11 & Sclerosing stromal tumour \\
\hline Ghanbari 2013 [41] & 4 & 1 & 3 & Juvenile granulosa cell tumour \\
\hline Tsuboyama et al 2018 [42] & 4 & 2 & $14(1), 10(2)$ & Dysgerminoma \\
\hline Bedir et al 2014 [43] & 4 & 1 & 10 & Juvenile granulosa cell tumour \\
\hline Boraschi et al 2008 [44] & 4 & 1 & 7 & Immature teratoma \\
\hline Chaurasia et al 2014 [45] & 4 & 1 & 7 & Sclerosing stromal tumour \\
\hline Lin et al 2017 [46] & 4 & 74 & 6 & Germ cell tumours \\
\hline Pollmann et al 2017 [47] & 4 & 1 & 13 & Mature teratoma \\
\hline Braun et al 2012 [48] & 4 & 1 & 12 & Leydig cell tumour \\
\hline Calcaterra et al 2013 [49] & 4 & 1 & 8 & Juvenile granulosa cell tumour \\
\hline Rogers et al 2014 [50] & 4 & 129 & 12 & Benign and malignant \\
\hline Nejkovic et al 2012 [51] & 4 & 1 & 17 & Mature teratoma \\
\hline
\end{tabular}

Characteristics of all studies included in this systematic review, including the number of ovarian masses analysed, histopathological classification hereof and mean age of the participants per concerning study. The methodologic quality of included studies based on the Oxford Centre for Evidence-Based Medicine Levels of Evidence Classification rubric is provided as well

$n=$ population size expressed as number of ovarian masses included in the original study

$N A$, not available

significant overlap, with values for benign masses varying between 1.16 and $2.03 \times 10^{-3} \mathrm{~mm}^{2} / \mathrm{s}$, whereas the range of ADC values reported for malignant masses was 0.76 to $1.39 \times 10^{-3} \mathrm{~mm}^{2} / \mathrm{s}$. Three of the included studies provided information on the diagnostic performance of DWI $[23,25,31]$. Nasr et al provided diagnostic performance of DWI solely, with sensitivity, specificity and accuracy of $100 \%, 75 \%$ and $87 \%$ respectively [23]. Emad-Eldin et al and Mansour et al demonstrated sensitivity, specificity and accuracy of DWI additional to MR imaging of $100 \%$ and $93.3 \%$; $96.77 \%$ and $85 \%$; and $98.46 \%$ and $82.3 \%$ respectively [25, 31]. The diagnostic performance of specific ADC cut-off values, if provided, is shown in Table 4 . 


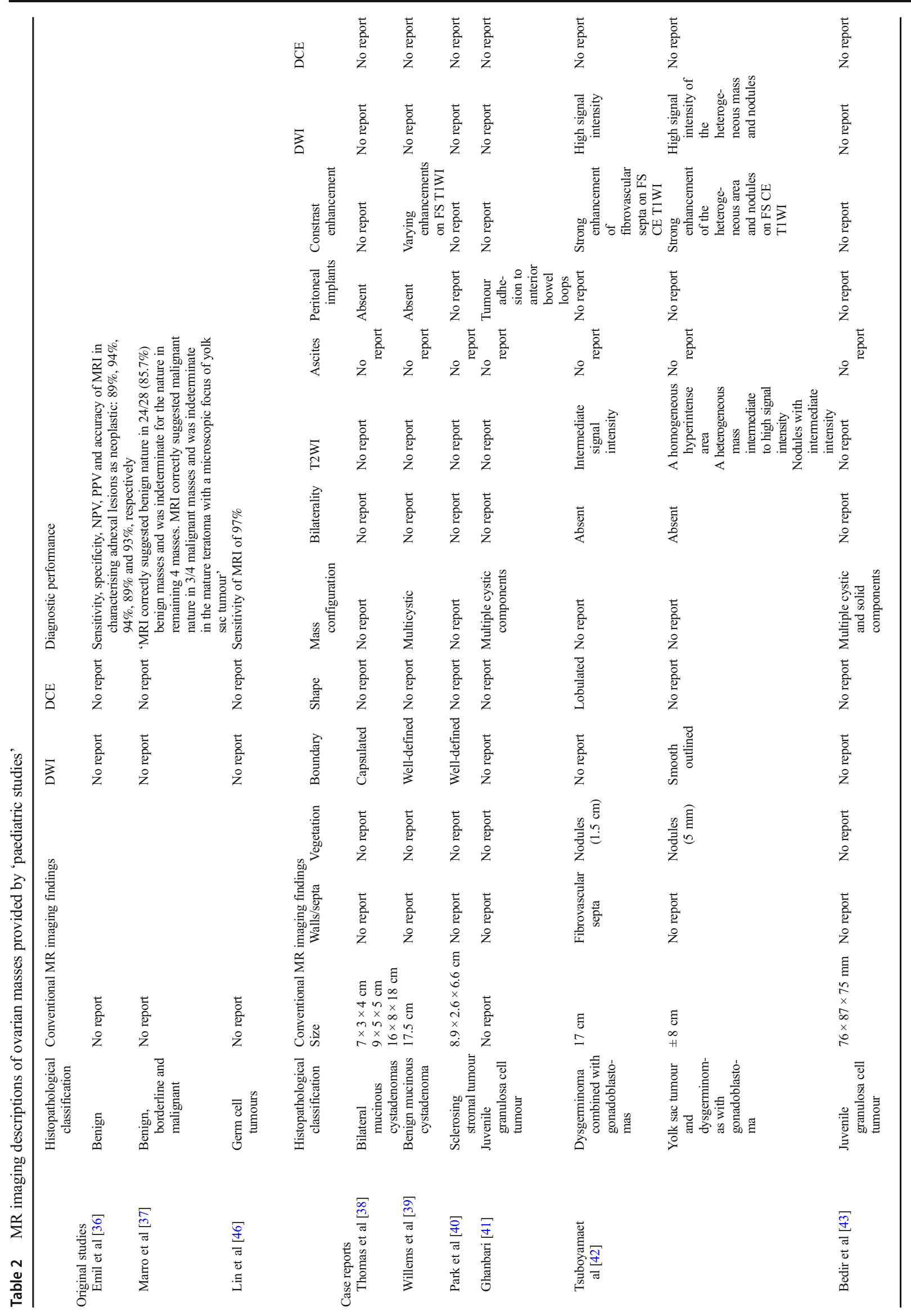




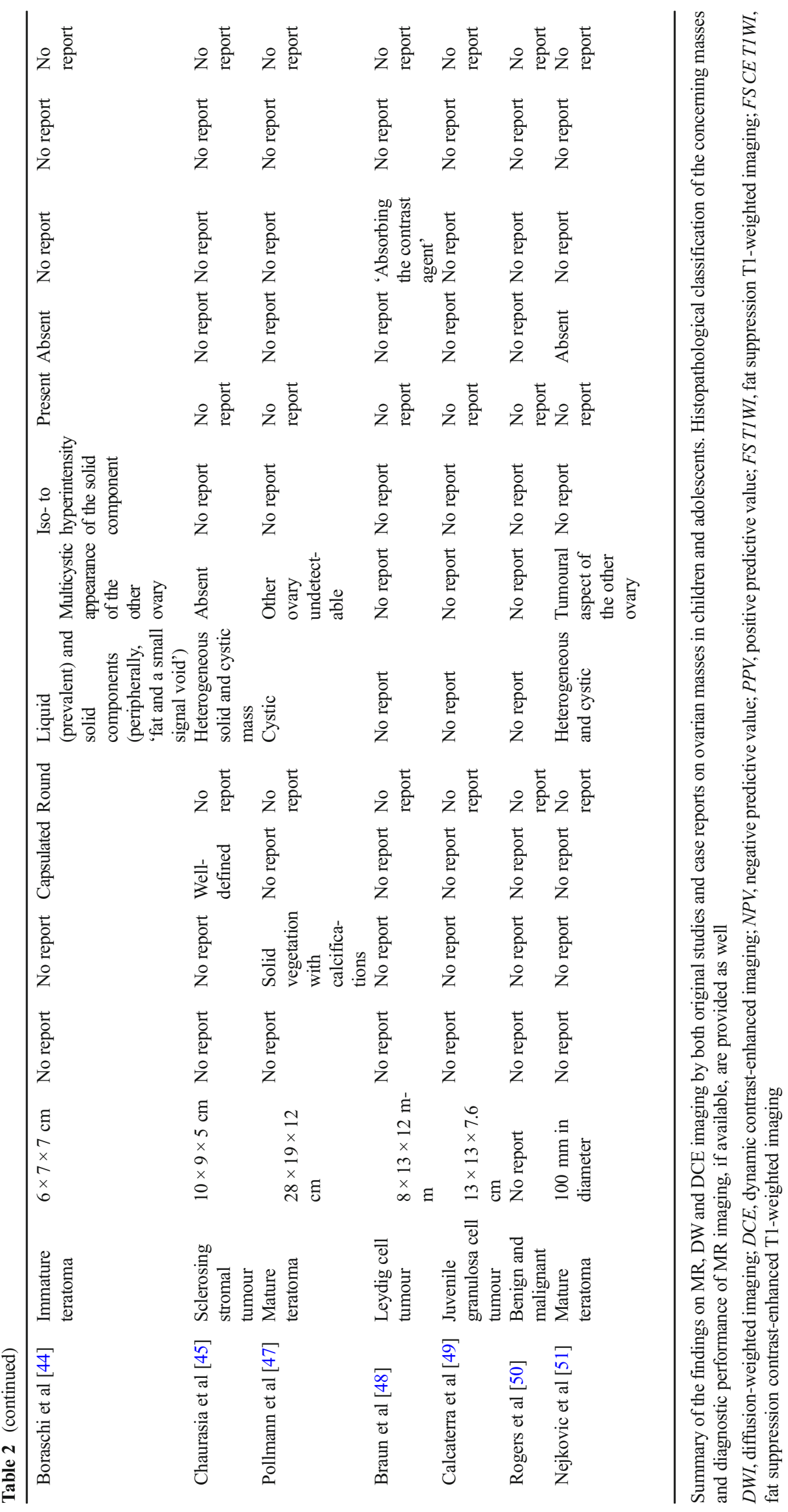




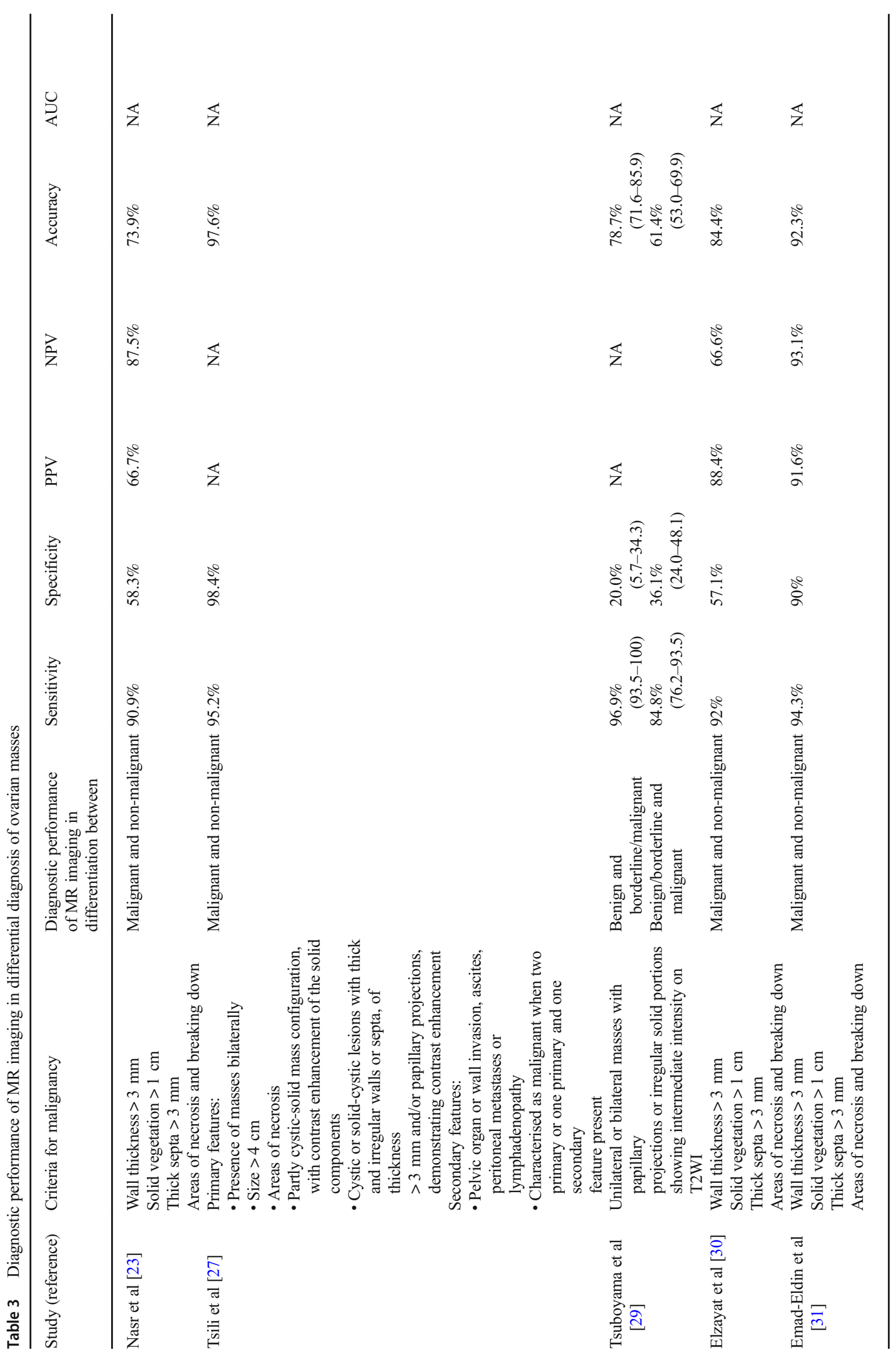





$85.7 \%$ of benign masses showing type I TICs. In type III TICs,
Nine studies investigated the value of DCE-MRI in the differential diagnosis of ovarian masses [11, 12, 22, 23, 25, 28, 30-32]. Data on the TICs and semi-quantitative DCE parameters are depicted in Table 5, as well as diagnostic performance of this sequence and accompanying TICs. Five of these studies divided the different ovarian masses analysed by type of TIC. Type I TICs were most frequently found in benign lesions, with 33 to on the other hand, there appeared more characteristics of malignancy, with 57.1 to $94.3 \%$ of all malignant masses exhibiting type III TICs. Overlap between benign and malignant masses was found by Elzayat et al [30] and Mansour et al [32], with one and nine malignant masses exhibiting a type I TIC, respecbenign masses exhibiting a type III TIC. The enhancement amplitude constituted one of the semi-quantitative parameters and hancent percentage (MRE\%), maximum absolute enhancement (SImax), maximum relative enhancement (SIrel) and signal intensity at $60 \mathrm{~s}$ after enhancement $\left(\mathrm{SI}_{60}\right)$. Malignant masses generally showed an increased enhancement amplitude compared with benign or borderline masses, with some of the studies demonstrating a statistically significant difference between these groups. Time to peak constituted the other semi-quantitative parameter and was indicated by time of half rising (THR), Tmax and time to peak within $200 \mathrm{~s}$ after enhancement $\left(\mathrm{TTP}_{200}\right)$. All studies analysing this parameter agreed on malignant masses exhibiting a shorter time to peak compared with benign masses, again in several of these studies with statistically significant difference. Four studies provided information on the diagnostic performance of DCE [23, 25, 30, 31]. Nasr et al [23] and Elzayat et al [30] provided diagnostic performance of solely DCE, with sensitivity, specificity and accuracy of $60 \%$ and $80 \% ; 91 \%$ and 100\%; and 77.2\% and 96\%, respectively. Mansour et al [25] and Emad-Eldin et al [31] demonstrated sensitivity, specificity and 100 and $100 \%$; and $95 \%$ and $96.9 \%$, respectively.

\section{Discussion}

Pre-operative discrimination between benign and malignant ovarian masses is of major importance, particularly in children and adolescents, where preserving fertility constitutes a highly important aspect of the therapeutic approach. Although data of MR imaging from paediatric patients were scarce, this review suggests that DWI, with ADC values measured in enhancing components, and semi-quantitative DCE might increase the diagnostic performance of MR imaging in the pre-operative differentiation between benign and malignant ovarian masses. 












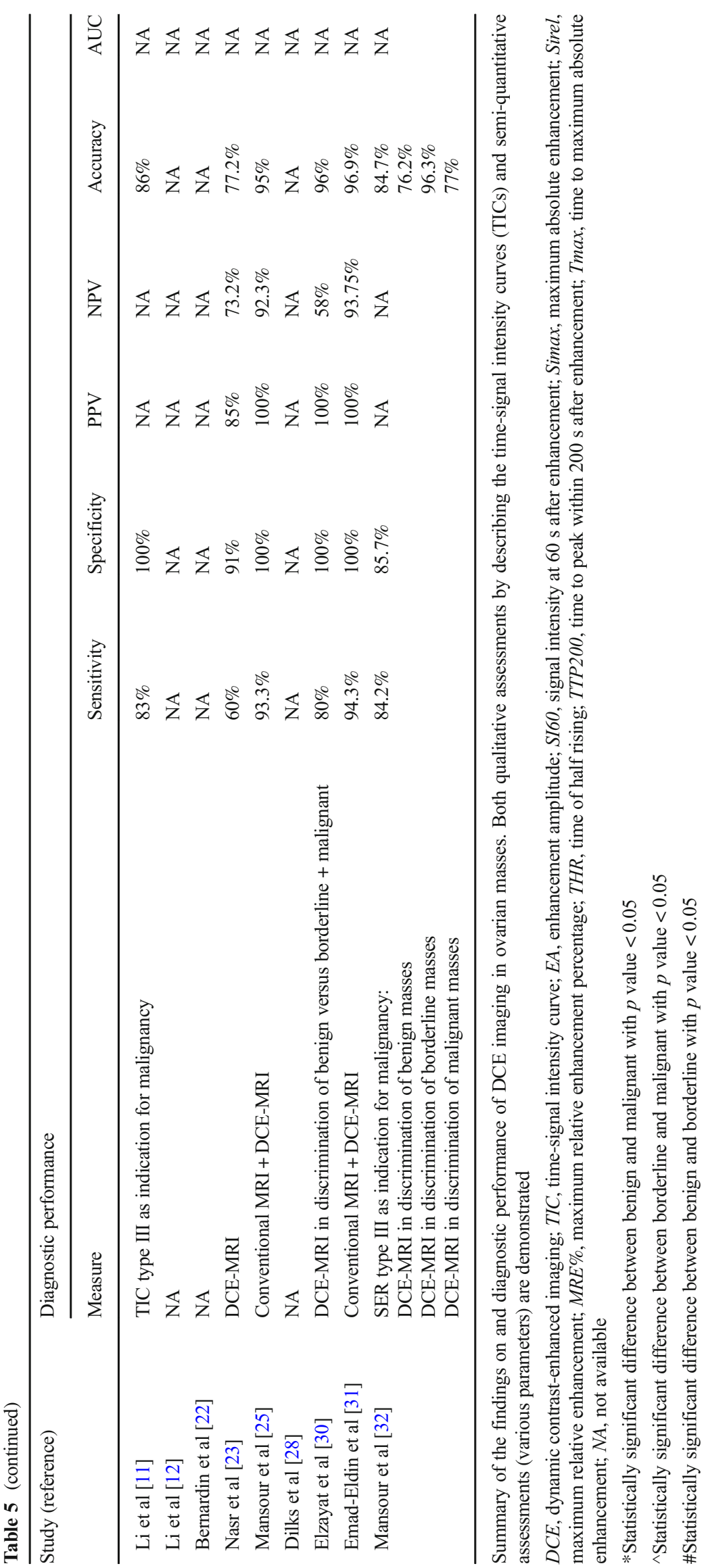


MR imaging characteristics associated with malignancy included larger size, thicker walls, presence of septa and/or vegetation within the mass, increased signal intensity on T2-weighted imaging, increased contrast enhancement, ascites, peritoneal implants and bilaterality. This corresponds with reports in existing literature describing masses larger than $4 \mathrm{~cm}$, with solid components demonstrating contrast enhancement or cystic lesions with vegetation $>1 \mathrm{~cm}$ (as profuse papillary projections), wall and septum thickness of $>3 \mathrm{~mm}$ and areas of necrosis as suspicious [52-54]. Diagnostic performance of MR imaging has a fairly good sensitivity for differentiating malignant from benign masses. Regarding specificity, however, there is still room for improvement.

DWI seems to improve sensitivity and specificity of MR imaging to $93.3-100 \%$ and $85-96.8 \%$, respectively [25, 31]. The added value of ADC is less clear. Although ADC values for malignant masses were lower compared with benign tumours, a considerable overlap was found. This can partly be explained by ADC values depending strongly on the pathologies included, the $b$-values used and whether ADC is calculated on both solid and cystic components of the lesion, or solely solid components. Several masses of benign origin, including mature teratomas, cystic endometriosis and fibromas, might occur as false positives. These 'complex masses' have a more dense composition, not as a result of increased cellularity but rather as a result of the presence of keratinoid substances, products of haemoglobin degradation and dense fibres respectively [24, 25, 31]. To date, no consensus exists on which preferred $b$-value should be used in DWI of ovarian masses. When solely analysing the studies that focussed on 'complex masses' (excluding fat-containing lesions or solely cystic masses), using $b$-values of $>800 \mathrm{~s} / \mathrm{mm}^{2}$ and calculating ADC on solid components of the mass, considerably less overlap in ADC values was demonstrated [20, 24-26, 31]. Mean ADC values for benign masses then varied between 1.16 and $1.38 \times 10^{-3} \mathrm{~mm}^{2} / \mathrm{s}$ and for malignant masses between 0.76 and $1.03 \times 10^{-3} \mathrm{~mm}^{2} / \mathrm{s}$. DWI should be performed as an additional sequence in assessing non-fatty, non-haemorrhagic ovarian masses, with ADC values only measured in enhancing components of solid lesions, preferably with the highest $b$-value of $>800 \mathrm{~s} / \mathrm{mm}^{2}$ [7]. Additionally, our results suggest an ADC cutoff of $1.1 \times 10^{-3} \mathrm{~mm}^{2} / \mathrm{s}$ might represent the best cut-off to help discriminate between benign and malignant lesions.

Another sequence that might contribute to the specificity of MR imaging is based on the process of angiogenesis, which is characteristic of and essential to nearly all malignant tumours [11, 12]. DCE MR attempts to differentiate between benign, borderline and malignant masses by attributing them to one of the three TICs as obtained by DCE. This systematic review shows type I TICs to be fairly predictive of benign origin of the ovarian mass, whereas type III TICs are predictive of malignancy. However, the assessment of enhancement patterns remains qualitative and might therefore be subject to user bias, similar to the evaluation of masses based on morphological criteria [55]. The use of semi- quantitative parameters deducted from the TIC, for example the enhancement amplitude and time to peak, might offer a solution to this subjectivity. Unfortunately, no reliable cut-off values could be extracted due to much heterogeneity of the studies regarding the semi-quantitative parameters analysed and their corresponding cut-off values as well as diagnostic performance. TIC type alone might not be sufficient in distinguishing between benign and malignant masses, since malignant lesions such as adenocarcinomas are sometimes found to be hypovascular, whereas benign masses, e.g. thecomas or sclerosing stromal tumours, might show hypervascularity [11]. Nevertheless, the diagnostic performance of the semi-quantitative parameters seems promising and DCE-MR imaging might thus form a valuable addition. We therefore support the advice of the ESUR to consider DCE-MR imaging in inhomogeneous solid masses on T2 or in complex cystic or cystic/solid masses with concern for malignancy. To deal with the aforementioned user bias and increasing extent of the diagnostic workup of ovarian masses (by incorporating DWI and DCE as well), there might be an interesting role for radiomics to play. This 'data-driven' approach which enables the extraction of innumerable quantitative features from tomographic images has already shown promising results in the classification of ovarian epithelial cancer, as well as in predicting several outcome measures [56, 57]. MR spectroscopy has also been reported to play a role in differentiating between borderline and malignant epithelial ovarian tumours [58]. However, epithelial tumours are rare in the paediatric population.

This systematic review faced some limitations. Data on the performance of MR imaging, combined with DWI and DCE, were largely deducted from studies performed in adult women (with no inclusion of paediatric patients), as MR imaging descriptions by paediatric studies were insufficient and no data from a purely paediatric cohort could be obtained. However, in order to minimise the risk of missing relevant studies, such studies and case reports in paediatric patients were included. The included studies showed much heterogeneity in MR imaging protocols, which made a meta-analysis impossible.

The description of the MR imaging features of the ovarian masses was very limited in the paediatric studies, which hampers the implementation for clinical use. Previously published reviews on the imaging of ovarian masses in children and adolescents were mainly based on findings in adult women [59-62]. This systematic review attempted to select studies applicable to children and adolescents, by exclusively including studies that were conducted either on paediatric patients or on adult women where at least $20 \%$ of the included patients had a malignant ovarian tumour other than carcinoma.

In conclusion, this systematic review suggests that DWI, with ADC values measured in enhancing components, and semi-quantitative DCE might further increase the diagnostic performance of MR imaging in the pre-operative differentiation between benign and malignant ovarian masses. Furthermore, our data show that an ADC cut-off of $1.1 \times$ 
$10^{-3} \mathrm{~mm}^{2} / \mathrm{s}$ might contribute to this differentiation. Prospective age-specific studies are needed to confirm the high diagnostic performance of MR imaging in combination with DWI and DCE techniques in children and adolescents with a sonographically indeterminate ovarian mass.

Funding information The authors state that this work has not received any funding.

\section{Compliance with ethical standards}

Guarantor The scientific guarantor of this publication is Dr. A.M.C. Mavinkurve-Groothuis.

Conflict of interest The authors of this manuscript declare no relationships with any companies, whose products or services may be related to the subject matter of the article.

Statistics and biometry No complex statistical methods were necessary for this paper.

Informed consent Written informed consent was not required for this study because this is a systematic review.

Ethical approval Institutional Review Board approval was not required because this is a systematic review.

Study subjects or cohorts overlap Some study subjects or cohorts have been previously reported in various articles (systematic review).

\section{Methodology}

- Systematic review

- Performed at one institution

Open Access This article is distributed under the terms of the Creative Commons Attribution 4.0 International License (http:// creativecommons.org/licenses/by/4.0/), which permits unrestricted use, distribution, and reproduction in any medium, provided you give appropriate credit to the original author(s) and the source, provide a link to the Creative Commons license, and indicate if changes were made.

\section{References}

1. Cancer Research UK Ovarian cancer incidence by age. In: Cancer Res UK https://www.cancerresearchuk.org/health-professional/ cancer-statistics/statistics-by-cancer-type/ovarian-cancer/ incidence\#heading-One. Accessed 1 Jan 2019

2. Kirkham YA, Lacy JA, Kives S, Allen L (2011) Characteristics and management of adnexal masses in a Canadian pediatric and adolescent population. J Obstet Gynaecol Can 33(9):935-943

3. Stanković ZB, Sedlecky K, Savić D, Lukač BJ, Mažibrada I, Perovic S (2017) Ovarian preservation from tumors and torsions in girls: prospective diagnostic study. J Pediatr Adolesc Gynecol 30(3):405-412

4. Hermans AJ, Kluivers KB, Janssen LM et al (2016) Adnexal masses in children, adolescents and women of reproductive age in the Netherlands: a nationwide population-based cohort study. Gynecol Oncol 143(1):93-97

5. Hermans AJ, Kluivers KB, Siebers AG et al (2016) The value of fine needle aspiration cytology diagnosis in ovarian masses in children and adolescents. Hum Reprod 31(6):1236-1240
6. Tavasolli F, Devilee P, World Health Organization Classification of Tumours (2003) Pathology and genetics of tumours of the breast and female genital organs. WHO Classification of Tumours, 3rd Edition, Volume 4

7. Forstner R, Thomassin-Naggara I, Cunha TM et al (2017) ESUR recommendations for MR imaging of the sonographically indeterminate adnexal mass: an update. Eur Radiol 27(6):2248-2257

8. Papic JC, Finnell SME, Slaven JE, Billmire DF, Rescorla FJ, Leys CM (2014) Predictors of ovarian malignancy in children: overcoming clinical barriers of ovarian preservation. J Pediatr Surg 49(1):144-148

9. Timmerman D, Testa AC, Bourne T et al (2008) Simple ultrasoundbased rules for the diagnosis of ovarian cancer. Ultrasound Obstet Gynecol 31(6):681-690

10. Thomassin-Naggara I, Daraï E, Cuenod CA et al (2009) Contribution of diffusion-weighted MR imaging for predicting benignity of complex adnexal masses. Eur Radiol 19(6):1544-1552

11. Li HM, Qiang JW, Ma FH, Zhao SH (2017) The value of dynamic contrast-enhanced MRI in characterizing complex ovarian tumors. J Ovarian Res 10(1):4

12. Li X, Hu JL, Zhu LM et al (2015) The clinical value of dynamic contrast-enhanced MRI in differential diagnosis of malignant and benign ovarian lesions. Tumor Biol 36(7):5515-5522

13. Masch WR, Daye D, Lee SI (2017) MR imaging for incidental adnexal mass characterization. Magn Reson Imaging Clin N Am 25(3):521-543

14. Foti PV, Attinà G, Spadola $\mathrm{S}$ et al (2016) MR imaging of ovarian masses: classification and differential diagnosis. Insights Imaging $7(1): 21-41$

15. Vargas HA, Barrett T, Sala E (2013) MRI of ovarian masses. J Magn Reson Imaging 37(2):265-281

16. Mohaghegh P, Rockall AG (2012) Imaging strategy for early ovarian cancer: characterization of adnexal masses with conventional and advanced imaging techniques. Radiographics 32(6):1751-1773

17. Moher D, Liberati A, Tetzlaff J, Altman DG, PRISMA Group (2009) Preferred reporting items for systematic reviews and metaanalyses: the PRISMA Statement. PLoS Med 6(7):e1000097

18. Bossuyt PM, Reitsma JB, Bruns DE et al (2015) STARD 2015: an updated list of essential items for reporting diagnostic accuracy studies. Clin Chem 61(12):1446-1452

19. Howick J, Chalmers I, Glasziou P et al (2011) The Oxford levels of evidence 2. Oxford Cent Evidence-Based Med 1:5653

20. Zhao SH, Li HM, Qiang JW, Wang DB,, Fan H (2018) The value of MRI for differentiating benign from malignant sex cord-stromal tumors of the ovary: emphasis on diffusion-weighted MR imaging. J Ovarian Res 11(1):73

21. Zhang H, Zhang GF, He ZY, Li ZY, Zhu M, Zhang GX (2012) Evaluation of primary adnexal masses by $3 \mathrm{~T}$ MRI: categorization with conventional MR imaging and diffusion-weighted imaging. J Ovarian Res 5(1):1-8

22. Bernardin L, Dilks P, Liyanage S, Miquel ME, Sahdev A, Rockall A (2012) Effectiveness of semi-quantitative multiphase dynamic contrast-enhanced MRI as a predictor of malignancy in complex adnexal masses: radiological and pathological correlation. Eur Radiol 22(4):880-890

23. Nasr E, Hamed I, Abbas I, Khalifa NM (2014) Dynamic contrast enhanced MRI in correlation with diffusion weighted (DWI) MR for characterization of ovarian masses. Egypt J Radiol Nucl Med 45(3):975-985

24. Takeuchi M, Matsuzaki K, Nishitani H (2010) Diffusion-weighted magnetic resonance imaging of ovarian tumors: differentiation of benign and malignant solid components of ovarian masses. $\mathrm{J}$ Comput Assist Tomogr 34(2):173-176

25. Mansour S, Wessam R, Raafat M (2015) Diffusion-weighted magnetic resonance imaging in the assessment of ovarian masses with suspicious features: strengths and challenges. Egypt J Radiol Nucl Med 46(4):1279-1289 
26. Zhang P, Cui Y, Li W, Ren G, Chu C, Wu X (2012) Diagnostic accuracy of diffusion-weighted imaging with conventional MR imaging for differentiating complex solid and cystic ovarian tumors at 1.5T. World J Surg Oncol 10:237

27. Tsili AC, Tsampoulas C, Argyropoulou M et al (2008) Comparative evaluation of multidetector $\mathrm{CT}$ and MR imaging in the differentiation of adnexal masses. Eur Radiol 18(5):1049-1057

28. Dilks P, Narayanan P, Reznek R, Sahdev A, Rockall A (2010) Can quantitative dynamic contrast-enhanced MRI independently characterize an ovarian mass? Eur Radiol 20(9):2176-2183

29. Tsuboyama T, Tatsumi M, Onishi H et al (2014) Assessment of combination of contrast-enhanced magnetic resonance imaging and positron emission tomography/computed tomography for evaluation of ovarian masses. Invest Radiol 49(8):524-531

30. Elzayat WA, El-Kalioubie M, Abdel-Naby MM, Abdel-Malek RR (2017) The role of dynamic contrast enhanced MR imaging in the assessment of inconclusive ovarian masses. Egypt J Radiol Nucl Med 48(4):1159-1169

31. Emad-Eldin S, Grace MN, Wahba MH, Abdella RM (2018) The diagnostic potential of diffusion weighted and dynamic contrast enhanced MR imaging in the characterization of complex ovarian lesions. Egypt J Radiol Nucl Med 49(3):884-891

32. Mansour SM, Saraya S, El-Faissal Y (2015) Semi-quantitative contrast-enhanced MR analysis of indeterminate ovarian tumours: when to say malignancy? Br J Radiol 88(1053):20150099

33. Li H, Feng F, Qiang J et al (2018) Quantitative dynamic contrastenhanced MR imaging for differentiating benign, borderline, and malignant ovarian tumors. Abdom Radiol (NY) 43(11):3132-3141

34. Zhang H, Zhang GF, He ZY, Li ZY, Zhang GX (2014) Prospective evaluation of $3 \mathrm{~T}$ MRI findings for primary adnexal lesions and comparison with the final histological diagnosis. Arch Gynecol Obstet 289(2):357-364

35. Zhao SH, Qiang JW, Zhang GF, Wang SJ, Qiu HY, Wang L (2014) MRI in differentiating ovarian borderline from benign mucinous cystadenoma: pathological correlation. J Magn Reson Imaging 39(1):162-166

36. Emil S, Youssef F, Arbash G et al (2018) The utility ofmagnetic resonance imaging in the diagnosis and management of pediatric benign ovarian lesions. J Pediatr Surg 53:2013-2018

37. Marro A, Allen LM, Kives SL, Moineddin R, Chavhan GB (2016) Simulated impact of pelvic MRI in treatment planning for pediatric adnexal masses. Pediatr Radiol 46(9):1249-1257

38. Thomas RL, Carr BR, Ziadie MS, Wilson EE (2012) Bilateral mucinous cystadenomas and massive edema of the ovaries in a virilized adolescent girl. Obstet Gynecol 120(2 Pt 2):473-476

39. Willems RP, Slangen B, Busari JO (2012) Abdominal swelling in two teenage girls: two case reports of massive ovarian tumours in puberty. BMJ Case Rep. https://doi.org/10.1136/bcr.11.2011.5143

40. Park SM, Kim YN, Woo YJ et al (2011) A sclerosing stromal tumor of the ovary with masculinization in a premenarchal girl. Korean J Pediatr 54(5):224-227

41. Ghanbari Z (2013) Premature thelarche and precocious puberty in a three-year-old girl with granulosa cell tumor. Int J Women's Health Reproduction Sci 1(2):7

42. Tsuboyama T, Hori Y, Hori M et al (2018) Imaging findings of ovarian dysgerminoma with emphasis on multiplicity and vascular architecture: pathogenic implications. Abdom Radiol (NY) 43(7):1515-1523

43. Bedir R, Mürtezaoğlu AR, Calapoğlu AS, Șehitoğlu İ, Yurdakul C (2014) Advanced stage ovarian juvenile granuloza cell tumor causing acute abdomen: a case report. Arch Iran Med 17(9):645-648

44. Boraschi P, Donati F, Battaglia V (2008) Acute abdomen due to twisted ovarian immature teratoma in a 7-year-old girl. Pediatr Emerg Care 24(8):557-560
45. Chaurasia J, Afroz N, Maheshwari V, Naim M (2014) Sclerosing stromal tumour of the ovary presenting as precocious puberty: a rare neoplasm. BMJ Case Rep bcr2013201124

46. Lin X, Wu D, Zheng N, Xia Q, Han Y (2017) Gonadal germ cell tumors in children: a retrospective review of a 10 -year single-center experience. Medicine (Baltimore) 96(26):e7386

47. Pollmann N, van der Steeg HJJ, Semmekrot BA (2017) A girl looking pregnant. Ned Tijdschr Geneeskd 161:D967

48. Braun R, Peter A, Warmann S, Fuchs J, Binder G (2013) Fast intraoperative testosterone assay confirms the location of an ovarian virilizing tumor in a young girl. Horm Res Paediatr 79(2):110-113

49. Calcaterra V, Nakib G, Pelizzo G et al (2013) Central precocious puberty and granulosa cell ovarian tumor in an 8-year old female. Pediatr Rep 5(3):e13

50. Rogers EM, Casadiego Cubides G, Lacy J, Gerstle JT, Kives S, Allen L (2014) Preoperative risk stratification of adnexal masses: can we predict the optimal surgical management? J Pediatr Adolesc Gynecol 27(3):125-128

51. Nejkovic L, Pazin V, Dragojevic-Dikic S (2012) Carney complex and teratoma maturum ovarii-a case report. Eur J Gynaecol Oncol 33(6):672-674

52. Hricak H, Chen M, Coakley FV et al (2000) Complex adnexal masses: detection and characterization with MR imagingmultivariate analysis. Radiology 214(1):39-46

53. Valentini AL, Gui B, Miccò M et al (2012) Benign and suspicious ovarian masses-MR imaging criteria for characterization: pictorial review. J Oncol 2012:481806

54. Jun SE, Lee JM, Rha SE, Byun JY, Jung IJ, Hahn ST (2002) CT and MR imaging of ovarian tumors with emphasis on differential diagnosis. Radiographics 22:1305-1325

55. Kazerooni AF, Malek M, Haghighatkhah H et al (2017) Semiquantitative dynamic contrast-enhanced MRI for accurate classification of complex adnexal masses. J Magn Reson Imaging 45(2):418-427

56. Zhang H, Mao Y, Chen X et al (2019) Magnetic resonance imaging radiomics in categorizing ovarian masses and predicting clinical outcome: a preliminary study. Eur Radiol:3358-3371

57. Rizzo S, Botta F, Raimondi S et al (2018) Radiomics of high-grade serous ovarian cancer: association between quantitative CT features, residual tumour and disease progression within 12 months. Eur Radiol 28(11):4849-4859

58. Ma FH, Li YA, Liu J, Li HM, Zhang GF, Qiang JW (2018) Role of proton MR spectroscopy in the differentiation of borderline from malignant epithelial ovarian tumors: a preliminary study. J Magn Reson Imaging. https://doi.org/10.1002/jmri.26541

59. Pai DR, Ladino-Torres MF (2013) Magnetic resonance imaging of pediatric pelvic masses. Magn Reson Imaging Clin N Am 21(4): 751-772

60. Heo SH, Kim WJ, Shin SS et al (2014) Review of ovarian tumors in children and adolescents: radiologic-pathologic correlation 1. Radiographics 34(5):2039-2055

61. Lam CZ, Chavhan GB (2018) Magnetic resonance imaging of pediatric adnexal masses and mimics. Pediatr Radiol 48(9):12911306

62. Epelman M, Chikwava KR, Chauvin N, Servaes S (2011) Imaging of pediatric ovarian neoplasms. Pediatr Radiol 41(9):1085-1099

Publisher's note Springer Nature remains neutral with regard to jurisdictional claims in published maps and institutional affiliations. 